\title{
ON THE PERFORMANCE OF THE "6-3" ELEMENT IN CONSOLDATION ANALYSIS
}

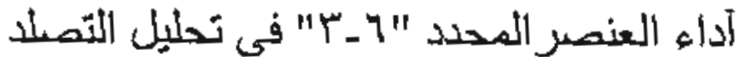

Dr. Baher L. Aboustait and Dr. Osama A. Mansour

Construction Engineering Department, Mise University for Science \& Tecbnology (Must), Cairo Egypt

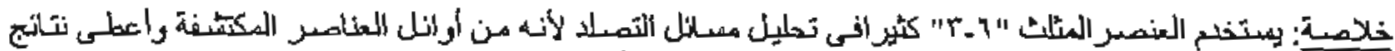

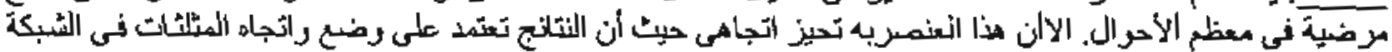

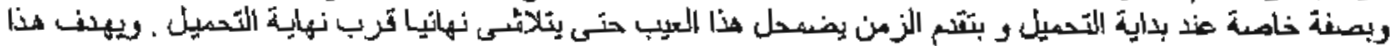

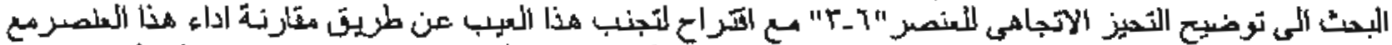

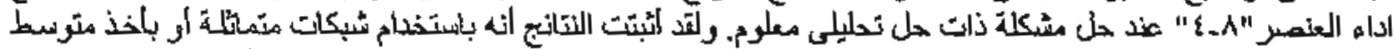

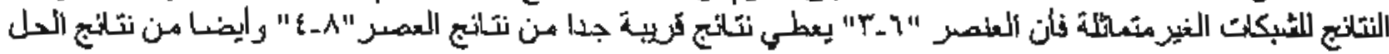

اللتحليلي.

\section{ABSTRACT}

The "6-3" triangular element has been widely used in consolidation analysis because it was the first one to be introduced and gave satisfactory results in most cases. Yet, it has a directional bias as the transient response depends on the orientation of the triangles in the mesh, particularly at the early stages of loading. As the time elapses this defect reduces until almost vanishes by the end of the loading history. The purpose of the present jnvestigation was to illustrate the directional bias of the "6-3" element and provide a solution to this problem by comparing its performance with that of the " $8-4$ " element in solving a problem with known analytical solution. It was found that by averaging the solution of the "6-3" element for non symmetric mesh or by using symmetric mesh, the "6-3" element provides a solution very close to that given by the " $8-4$ " element as well as the aralytical solution.

Keywords: Consolidation, Variational formulations, Finite element discretization

\section{INTRODUCTION}

Sandhu and Wilson [1, 2] presented the first application of the finite element method to analysis of seepage in elastic media. Considerable progress has been made in the theoretical formulation as well as computational procedures. This includes variational formulations admitting linited smoothness of finite element bases $[3,4]$ and experimentation with several different spatial interpolation schemes and investigation of various temporal approximation methods [511]. The finite element method has been applied to saturated soils exhibiting secondary compression $[7,10]$, nonlinear soil behavior [11-13], and to finite deformation [12]. The method has been extended by Aboustait $[14,15]$ to formulate the coupled theories of thermoelastic and thermoplastic consolidation and the associated variational principles. Further, the formulation was extended to include inertia and damping effects resulting in finite element Galerkin formulation for a dynamic consolidation theory by Aboustait and
Sandhu [16, 17]. Least-Squares mixed finite element formulation is presented for Biot's consolidation using piecewise linear and quadratic interpolation for the fluid pressure and for the displacement, respectively [18]. Very Recently new approaches have been advanced based on mixed formulations. A fully coupled 3-D mixed finite element model is developed with the aim at alleviating the pore pressure numerical oscillations at the interface between materials with different permeability [19]. In spatial discretization, Sandhu [1] proposed that the order of terms appearing in a convolution product in the variational principle be the same. This produced the "composite" element in which the order of polynomial interpolation for displacements was higher than that for fluid pressures. The composite element first proposed by Sandhu $[1,2]$ and used by Hwang [20] and others, was the "6-3" element with quadratic interpolation for displacements and linear 


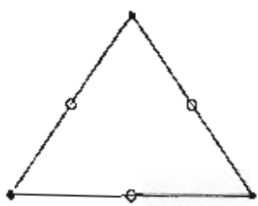

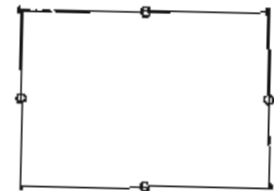

Nodes with $(u, v, \pi)$

Nodes with $(u, v)$

a) The " $6-3$ " Composite Elcment

b) The "8-4" Composite Element

Figare (1): The "6-3" and "8-4" Composite Elements

interpolation for fluid pressures over triangular regions. Later, Sandhu [6] introduced the " $8-4$ " element which had eight point biquadratic interpolations for displacements and four point isoperimetric quadrilateral for fluid pressures. Figure (1) illustrates the"6-3"and the "8-4" elements. Later this element was extended by Aboustait $[14,15]$ to obtain the "8-4-4" element which includes another four point isoperimetric quadrilateral interpolations for temperature fields. It is used in solving thermoelastic and thermoplastic consolidation problems. Several spatial interpolation schemes, besides the composite elements, have been tried by various investigators. Ghaboussi [5] introduced the "6-4" element which uses four point isoparametric quadrilaterals for both fields. However, two additional incompatible modes were included in the displacement approximation. This element has the economy while the additional "local" mode gives-it the character of a "higher order" scheme. Smith [21] presented the "4-4" element and the formulation was similar to Ghaboussi's except that no incompatible modes were used. Prevost [9] proposed cautious use of "reduced integration" in conjunction with Smith's "4-4" element. Yokoo [22], Booker [23], and Vermeer [24] used triangular elements with linear interpolation for both displacement and fuid pressure fields, i.e., "3-3" element.

All investigators have generally reported success with whatever scheme they used. Comparative studies of different elements are rare. Some comparisons between numerical performances of the "6-3" and the "8-4" elements were attempted by Sandhu et al $[6,7]$ and between the "6-4"and the "8-4" elements by Aboustait et al [25, 26]. In evaluating yarious candidate schemes, $[6,7]$ proposed that an acceptable nethod meet the following requirements in addition to efficiency and accuracy;

$i$. The interpolation scheme must conform to the assumptions regarding continuity as well as differentiability used in setting up the governing variational formulation.

ii. It should be possible to generate the "undrained" solution, i.e. the state of fluid pressures and displacement at time $t=0+$.

iii. For sufficiently small time steps, the scheme should be insensitive to the choice of the timestep size.

Elements "6-3", "6-4" and "8-4" satisfy these previous requirements. However, the "6-3" and "8-4" composite elements are too expensive to be used in large problems. This has discouraged extension of the analysis to three-dimensions, and to nonlinear analysis and dynamic problems. The "4-4" element is more economical but has oscillatory errors [7]. The "6-3" element gives satisfactory results in most cases and the "8-4" element gives results almost identical to those from the "6-3" element but is more economical as it requires fewer nodal points and has less bandwidth $[6,7]$. A comparison between the " $6-4$ " and " $8-4$ " elements was carried by Aboustait $e t$ al $[25,26]$ in solving Terzaghi and Gibson problems. The " $6-4$ " element gave a solution identical to that given by the "84"element but with significant savings in computational time. Also, it is distinctly superior to the " $4-4$ " element as it does not have the oscillatory error of the "44 " element. Further, it directly gives the solution at time $=0+$. This is because, in eliminating the additional degrees of freedom, the static condensation would, in general, result in nonzero diagonal quantities. At the same time, it has the economy of the simpler element.

The "6-3" triangular element still has been widely used particularly in commercial packages. Yet, it has the defect of directional bias since the transient response depends on the orientation of the triangles in the mesh.

The purpose of the present investigation was to illustrate the directional bias of the "6-3" element 
and provide a solution to this problem. This had been achieved by performing another comparison between the performance of the "6-3"and the" 8 4" elements in solving the same Terzaghi's problem in $[6,7]$. Same boundary conditions, same loading, same material properties and same spatial and temporal discretizations were considered. However more attention was given to the transient response particularly at the early stages of loadings. Plane strain formulation is considered for both elements to solve Terzaghi's problem of one-dimensional consolidation that has well known analytical solution.

\section{EQUATIONS GOVERNING LINEAR ELASTIC SOIL CONSOLDATION}

Assuming pore water to be incompressible, the equation of force equilibrium of elementary volumes, within the spatial region of interest $R$, may be written in standard indicial notation as, [1];

$$
\left[E_{\mathrm{k}, y} u_{\mathrm{k}, j}\right]_{, 1}+\pi, j+o f_{j}=0
$$

Also, the mass continuity equation over that region can be expressed as;

$$
\left[K_{i j}\left(\pi_{y i}+\rho_{2} f_{i}\right)\right]_{, j}+\dot{u}_{j, j}=0
$$

i.e., the out flow from any closed surface equals to the rate of volume reduction. Where $u_{i}, f_{i j} E_{k l i j}$, $\mathrm{K}_{\mathrm{ij},}$ respectively denote the Cartesian components of the displacement vector, the body force vector per unit mass, the isothermal elasticity tensor and the permeability tensor, $\rho$ is the mass density of the saturated soil and $\rho_{2}$ is that of water, $\pi$ is the pore water pressure. With these field equations we associate the following boundary conditions;

$$
\begin{array}{ll}
u_{i}=\hat{u}_{i} & \text { on } S_{1} \\
t_{i}=\tau_{j j} n_{j}=\hat{i}, & \text { on } S_{2} \\
\pi=\hat{\pi} & \text { on } S_{3} \\
Q=q_{i} n_{j}=\hat{Q} & \text { on } S_{4}
\end{array}
$$

Here, $t_{i}$ and $q_{i}$ are components of the traction and fluid flux vectors associated with surfaces embedded in the closure of $R . S_{1}$ and $S_{2}$ are complementary subsets of the boundary of the spatial region of interest and so are $S_{3}, S_{1}$. $\tau_{i j}$ are

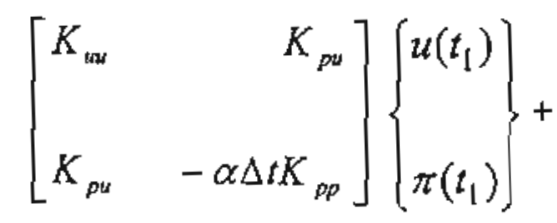

components of the total stress tensor which is related to the effective stress tensor $\sigma_{i j}$ and pore pressure by;

$$
\tau_{y j}=\sigma_{i j}+\pi \delta_{l j}
$$

$\delta_{i j}$ is the Kronecker delta. Noting that the effective stress tensor is related to the solid deformation $\varepsilon_{i \mathrm{j}}$ by;

$$
\sigma_{i j}=E_{i j k t^{\prime}} \varepsilon_{k t}
$$

The initial conditions for the problem are

$$
\begin{array}{ll}
u_{i}\left(x_{i}, o\right)=u_{i}(o) & \text { on } R \\
\pi\left(x_{j}, 0\right)=\pi(0) & \text { on } R
\end{array}
$$

The variational principle corresponding to a the above mentioned field equations, boundary and initial conditions can be expressed as;

$$
\begin{aligned}
& J(u, \pi)=\int_{v}\left[\frac{1}{2} \sigma_{b j}^{*} \varepsilon_{l j}-\phi_{i}^{*} u_{i}+\pi^{*} u_{i j}\right. \\
& \left.-\frac{1}{2} g^{*} q_{i}^{*}\left(\pi,+\rho_{2} f_{i}\right)\right] d v \\
& -\int_{s_{2}} \hat{t}_{i}^{*} u_{i} d s-\int_{s_{t}} \hat{Q}^{*} g^{*} \pi d s
\end{aligned}
$$

Where $g=1$ and $*$ is indication of convolution. $\hat{Q}$ and $\hat{t}_{j}$ are the prescribed fluid flow and tractions on $S_{2}$ and $S_{4}$ respectively.

\section{FINTE ELEMENT FORMULATION:}

Discretization of the governing functional for the two-field formulation followed by application of the variational principle (Ii) leads to the following matrix equation.

$$
\left\{\begin{array}{c}
-K_{p u} u\left(t_{0}\right) \\
(1-\alpha) \Delta t K_{p p} \pi\left(t_{0}\right)
\end{array}\right\}=\left\{\begin{array}{l}
p_{1} \\
p_{2}
\end{array}\right\}
$$


Where $\left(t_{0}, t_{1}\right)$ is the single time step of interest, and;

$\Delta t=t_{1}-t_{0}$

$\left\{u\left(t_{1}\right)\right\},\left\{u\left(t_{0}\right)\right\}=$ vectors of nodal point values of the components of the displacement at time $t_{1}, t_{0}$, respectively

$\left\{\pi\left(t_{0}\right)\right\},\left\{\pi\left(t_{0}\right)\right\}=$ vectors of nodal point values of the pore water pressure at time $t_{1}, t_{0}$, respectively.

$\left\{p_{1}\right\}=$ the vector of nodal point loads including applied nodal loads, boundary tractions, body forces, initial stresses and effect of displacement constraints .

$\left\{p_{2}\right\}=$ the vector of nodal point fluxes including applied nodal fluxes, boundary fluxes,

body force effects and effects of specified pore water pressures.

$\left[\mathrm{K}_{\mathrm{uu}}\right]=$ spatial "stiffness matrix" for the elastic soil.

$\left[\mathrm{K}_{\mathrm{pp}}\right]=$ the spatial "flow matrix" for the compressible fluid and $\Delta t=1$.

$\alpha=$ the coefficient characterizing single-step temporal discretization.

$\left[\mathrm{K}_{\mathrm{pu}}\right]=$ the coupling matrix representing the influence of pore pressure in the force equilibrium equation.

$\left[k_{\text {pu }}\right]^{T}=$ the coupling matrix representing the influence of soil volume change upon the noda] point fluid pressure.

$\left[\mathrm{K}_{\mathrm{pp}}\right]=$ the spatial fluid compressibility matrix.

The matrix $\left\{K_{\mathrm{uu}}\right\}$ depends upon the interpolation scheme for displacements and $\left[\mathrm{K}_{\mathrm{ppo}}\right]$ depend upon the interpolation scheme for the pore water pressures. The coupling matrix $\left[K_{\text {pu }}\right]$ involves spatial interpolation for both the field variables. The temporal discretization for the single step scheme is reflected in the value of the coefficiento. For linear interpolation $\alpha=0.5$.

Equation (12) includes the "natura!!" boundary conditions expressed by Equation (4) and (6). Equation (3) and (5) are satisfied by explicitly requiring $\omega_{1}=\hat{u}_{1}$ on $S_{1}$ and $\pi=\hat{\pi}$ on $S_{3}$.
Development of the vectors and matrices appearing in Equation (12) is given in [25].

\section{Terzaghi's problem of one-dimensional consolidation}

A computer program was developed for plane strain consolidation using both " $8-4$ " and " $6-3$ " elements. The code was used to solve Terzaghi's problem of one-dimensional consolidation. For this problen, the theoretical solutions are known and, therefore, precise comparison was possible.

For Terzaghi's problem, the dimensions of the consolidating soil column and soil properties were the same as in $[6,7,23,24]$; i.e. soil depth $h$ $=7 \mathrm{~m}$, modulus of elasticity $\mathrm{E}=6000 \mathrm{t} / \mathrm{m}^{2}$, Poisson's ratio $=0.4$, coefficient of permeability $\mathrm{K}=4 \times 10^{-6} \mathrm{~m} / \mathrm{s}$. Figure (2) illustrates the geometry and the boundary conditions of the problem.

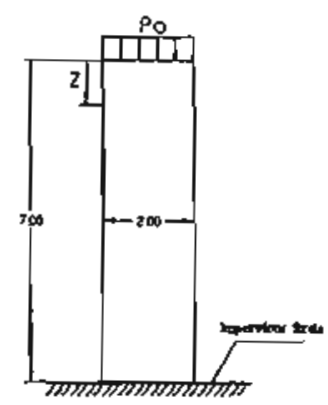

Figure (2): One dimensional Consolidation

Generally, finite element approximation involves discretization in the spatial as well as temporal domain. Accordingly, the investigation reported herein covered these two aspects. For the spatial discretizations, three different meshes were considered as shown in figure (3). Figure (3.a) and (3.6) illustrate biased and symmetric meshes that were used for the "6-3" element, while Figure (3.c) illustrates the mesh used for the "84 " element. The first mesh for the "6-3" element consisted of 18 elements with 57 nodes and the second mesh consisted of 36 elements with 84 nodes. While the "8-4" element mesh consisted of 9 elements with 48 nodes. 


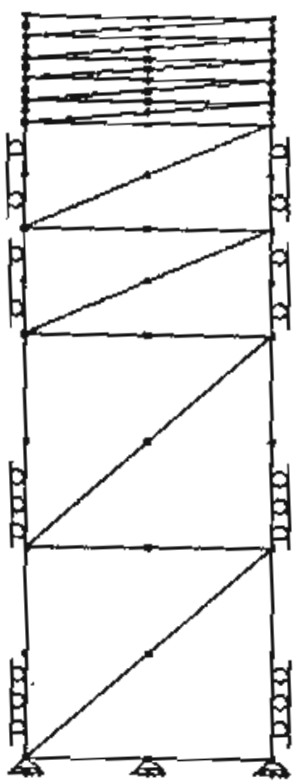

a) Non-Symmetric Mesh for the "6-3" Element

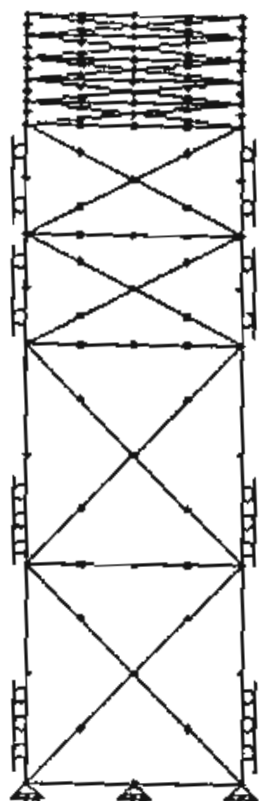

b) Symametric Mesh for the "6-3" Element

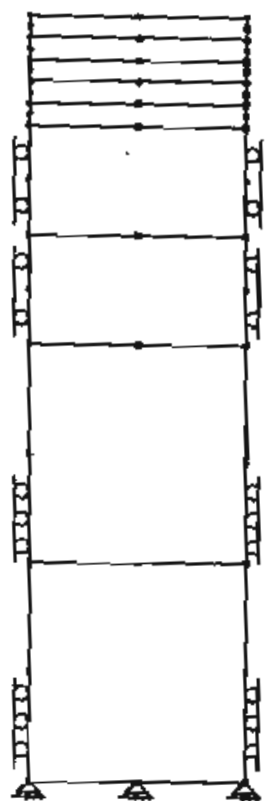

c) Mesh for the "8-4" Element

Figure (3): Finite Element Discretization

In the temporal discretization, linear interpolation was considered i.e., the coefficient $\alpha$ was given the value of 0.5 . For each of the spatial discretization schemes the following temporal partitioning was considered starting with $t=0$.

$$
\begin{array}{lr}
10 \text { steps of } \Delta t=0.01 & \text { over }[0,0.1] \\
10 \text { steps of } \Delta t=0.1 & \text { over }[0.1,1.1] \\
10 \text { steps of } \Delta t=10 & \text { over }[1.1,101.1] \\
10 \text { steps of } \Delta t=100 & \text { over }[101.1,1101.1] \\
8 \text { steps of } \Delta t=1000 & \text { over }[1101.1,9101.1]
\end{array}
$$

Where, the time is in seconds. In this scheme $\Delta$ changes in a ratio $10: 1$ except that the change from $\Delta t=0.1$ to $\Delta t=10$ has a ratio of 100:1.

\section{RESULTS OF ANALYSIS}

Table 1 shows the time settlement history for the three types of discretizations as well as the analytical solution. The settlements whicb are the vertical displacements of the two corner nodes on the top surface of the soil column were recorded. For the "6-3" element with non-symmetric mesh, the settlements of these two nodes are completely different at the early stages of loading, while they are the same for the other two meshes. As the time elapses, this difference decreases until it vanishes near the end of the loading history. Meanwhile the average settlement of these two nodes in the non-symmetric mesh for the "6-3" element practically coincided with the settlement of the symmetric mesh for the "6-3" element as well as response of the " $8-4$ " element throughout the time domain and also was in good agreement with the analytical solution. Overestimates of early settlement are associated with all spatial discretizations schemes considered. Also, it was found that the surface settlement calculated is insensitive to the temporal discretizations and the sudden change in the size of $\Delta t$. 
Table (1): Surface Settlement History

\begin{tabular}{|c|c|c|c|c|c|c|}
\hline \multirow{2}{*}{ The (sec) } & \multirow{2}{*}{$\begin{array}{l}\text { Symmetric Mesh } \\
\text { for the "6-3" } \\
\text { Element }\end{array}$} & \multicolumn{3}{|c|}{ Non-Symmetric Mesh for the "6-3" Element } & \multirow{2}{*}{$\begin{array}{l}\text { Element } \\
" 8-4{ }^{4}\end{array}$} & \multirow{2}{*}{ Exact Sohutlon } \\
\hline & & Left Node & Right Node & Average & & \\
\hline 0.02 & $4.6412 \mathrm{E}-06$ & $2.7936 E+06$ & $7,0104 \mathrm{E}-06$ & $4,90205-06$ & $5.2770 E-06$ & $5.7255 E-06$ \\
\hline 0.6 & $1.5091 E-05$ & $1.6537 \mathrm{E}-05$ & $1.3973 \mathrm{E}-05$ & $1.5255 \mathrm{E}-\mathrm{OS}$ & $1.5681 E-05$ & $1.5459 \mathrm{E}-05$ \\
\hline 1.1 & $2,0646 E-05$ & $2.1661 E-05$ & $1.9801 E-05$ & $2,0731 \mathrm{E}-05$ & $2.1221 \mathrm{E}-05$ & 20877 E.05 \\
\hline 21.1 & $8.1150 E-05$ & B.0815E-05 & 8.1492E-05 & B.1254E-05 & $9.1394 E-05$ & $9.1423 \mathrm{E}-05$ \\
\hline 41.1 & $1.2051 E-04$ & $1.2034 E-04$ & $1.2066 \mathrm{E}-04$ & $1.2050 E-04$ & $1.2815 E-04$ & $1.2760 E-04$ \\
\hline 81.1 & $1.7459 \mathrm{E}-04$ & $1.74506-04$ & $1.7467 \mathrm{E}-\mathrm{CA}$ & $1.7458 E-04$ & $1.8022 \mathrm{E}-04$ & 1.7924E-O4 \\
\hline 301.1 & $3.1443 E-04$ & $3.1440 E-04$ & $3.1446 E-04$ & $3.1443 \varepsilon-04$ & $3,4446 \mathrm{E}-04$ & $3.4205 \mathrm{E}-04$ \\
\hline 901.1 & $4.9727 \mathrm{E}-04$ & $4.9726 \mathrm{E}-04$ & $4.9727 \mathrm{E}-04$ & $4.9727 \mathrm{E}-04$ & $5.0352 \mathrm{E}-04 \quad 0$ & $5.0166 \mathrm{E}-04$ \\
\hline 2101.1 & $5.3400 \mathrm{E}-04$ & $5,3400 \mathrm{E}-04$ & $5.3400 E-04$ & $5.3400 \mathrm{E}-04$ & $5.4778 E-04$ & $5.4253 E-04$ \\
\hline 4101.1 & $5.4425 \mathrm{E}-04$ & 5.4425E-04 & $5.4425 E-04$ & $5.4425 \mathrm{E} \cdot \mathrm{OA}$ & $5,4456 \mathrm{E}-\mathrm{CO}$ & $5.4443 E-04$ \\
\hline $6101.1^{\circ}$ & $5.4444 E-04$ & $5.4444 E-04$ & 5.4444E- -04 & $5.4444 E-04$ & $5.4440 E-04$ & $5.4444 E-04$ \\
\hline 9101.1 & $5.4445 E-04$ & 5.4445E-0. & $5.4444 E-04$ & $5.4444 E-04$ & $5.4440 E-04$ & $5.4444 E-04$ \\
\hline
\end{tabular}

Table 2 and figure (4) show the pore pressure history for the three types of discretizations as well as the analytical solution. The pore pressure at the two corner nodes located at depth $=2 / 70$ of the total height, measured from the top surface of the soil column, were recorded. Same as for the surface settlement, the pore pressure at these nodes in the nonsymmetric mesh for the "6-3" are completely different at the early stages of loading, while they are the same for the other two meshes. As the time elapses, this difference decreases until vanishes near the end of the loading history. Also, the average pore pressure at these two nodes for the non-symmetric mesh for the "6-3" element practically coincided with the response of the symmetric mesh for the "63 " element as well as the "8-4" element throughout the time domain and also was in good agreement with the analytical solution, except at early time i.e. $t<0.1$. At early stages, the error in the pore pressure at points near the loaded surface is quite large for both the schemes. This is a feature of the spatial interpolation [7] used.

\section{CONCLUSIONS}

The "6-3" and " $8-4$ " elements were applied to Terzaghi's problem for which exact solution is available. Results of these limited tests show;

i.The "6-3" triargular element exhibit a directional bias and gave solution depends on the orientation of the triangle in the mesh particularly at early stages of loading. As the time elapses this defect reduces until completely vanishes at the end of the loading history

ii. The average solution of the "6-3" element for non-symmetric mesh or the solution of the "6-3"using symmetric mesh are identical to that given by the "8-4" element and the analytical solution.

iii. At very early stages of loading, both "6-3" and "8-4" elements gave unsatisfactory results. Apparently, special singularity elements are required near loaded drained surfaces. 
Table (2): Pore Pressure History

\begin{tabular}{|c|c|c|c|c|c|c|}
\hline \multirow[t]{2}{*}{$\begin{array}{l}\text { Time In } \\
\text { ands }\end{array}$} & \multirow{2}{*}{$\begin{array}{c}\text { "6-3" Element - } \\
\text { 5ymmetrl } \\
\text { Mesh }\end{array}$} & \multicolumn{3}{|c|}{ 6-3" Element - Nor-Symmetric Meth } & \multirow[t]{2}{*}{ "8-4" Element } & \multirow[t]{2}{*}{ Exact Solutlon } \\
\hline & & Left Node. & Plght Node & Average & & \\
\hline 0.02 & 1.035 & 1.423 & 1.009 & 1.216 & 1.217 & 0.987672331 \\
\hline 0.04 & 1,04 & 2.333 & 0.974 & 1.1535 & 1.156 & 0.962044728 \\
\hline 0.1 & 0.983 & 1.131 & 0.884 & 1.0075 & 1.0114 & 0.892813547 \\
\hline 0.6 & 0.597 & 0.618 & 0.569 & 0.5935 & 0.57489 & 0.575724899 \\
\hline 1.1 & 0.455 & 0.4696 & 0.4633 & 0.45345 & 0.45325 & 0,447440278 \\
\hline 21.1 & 0.102 & 0.099 & 0.1074 & 0.1032 & 0.20345 & 0.107914459 \\
\hline 41.1 & 0.0765 & 0.0749 & 0.0783 & 0.0766 & 0.076389 & 0.077436735 \\
\hline 61.1 & 0.064 & 0.0629 & 0.0651 & 0.064 & 0.063934 & 0.063543454 \\
\hline $\mathbf{g 1 . 1}$ & 0.0559 & 0.0552 & 0.0558 & 0.056 & 0.055978 & 0.055168022 \\
\hline 101.1 & 0.0502 & 0.0495 & 0.0509 & 0.05025 & 0.050273 & 0.049411265 \\
\hline 301.1 & 0.0299 & 0.0297 & 0.0302 & 0.02995 & 0.029943 & 0.026214407 \\
\hline 501.1 & 0.0177 & 0.0176 & 0.0179 & 0.01775 & 0.017772 & 0.015576324 \\
\hline 7011 & 0.0105 & 0.0104 & 0.0105 & 0.01045 & 0.010457 & 0.009272604 \\
\hline 901.1 & 0.0063 & 0.0061 & 0.0062 & 0.00615 & 0.0062456 & 0.005520156 \\
\hline 1101,1 & 0.0036 & 0.0036 & 0.00358 & 0.000359 & 0.0036 & 0.003286254 \\
\hline 9101.1 & 0.0036 & 0.0036 & 0,00358 & 0.00359 & 0.0036 & 0.003285254 \\
\hline
\end{tabular}

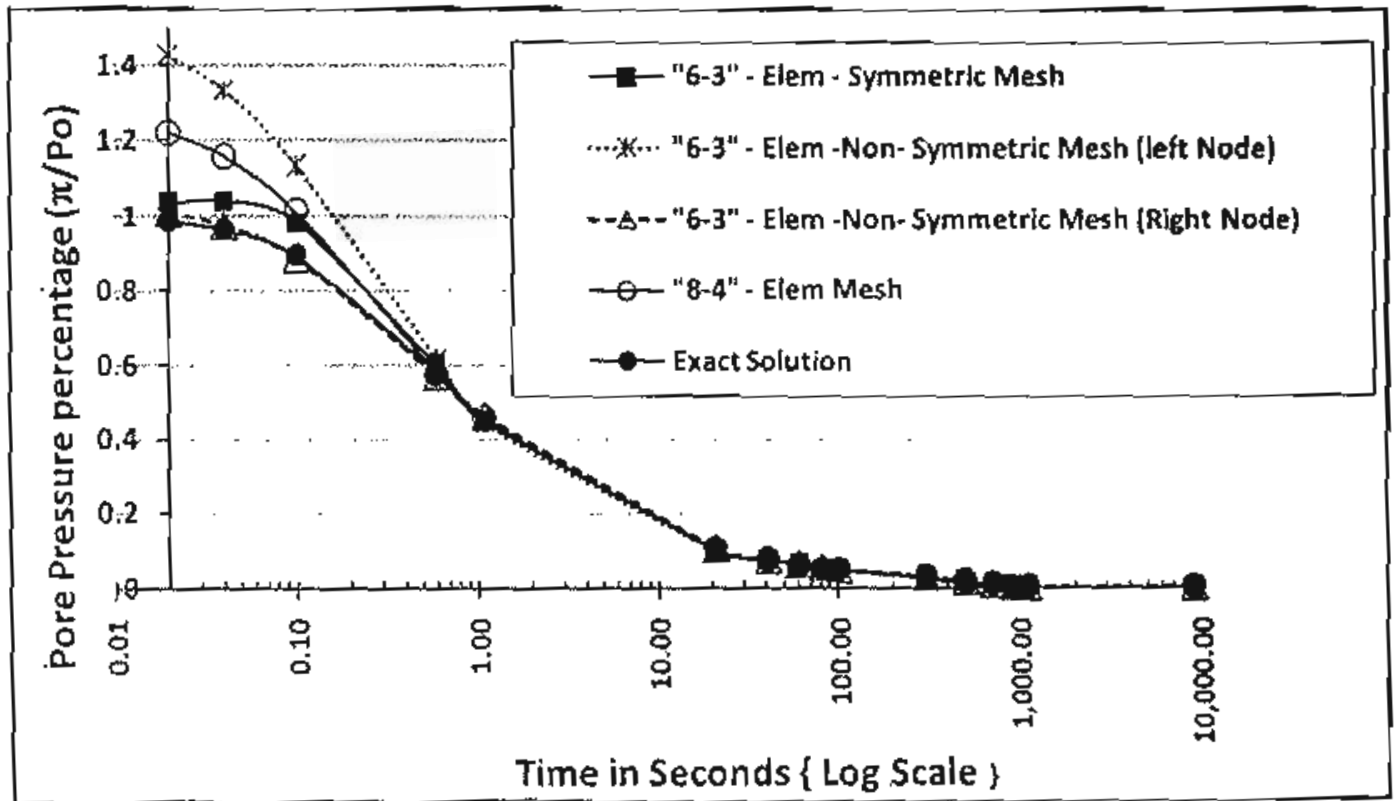

Figure (4): Pore Pressure Fistory 


\section{NOMENCLATURE}

$\mathrm{u}_{i} \quad$ the Cartesian components of the displacement vector

$\pi$ the pore water pressure

$f_{i}$ the Cartesian components of the body force vector per unit mass

$E_{\mathrm{k} \cdot \mathrm{ijj}}$ the isotherrnal elasticity tensor

$\mathrm{K}_{\mathrm{j} j}$ the permeability tensor

[N] the mass density of the saturated soil

$F(t)$ the mass density of water

$t_{i}$ the Cartesian components of the traction vector

$q_{i}$ the Cartesian components of the fluid flux vector

$\tau_{i j}$ the Cartesian components of the total stress tensor

$\sigma_{i j}$ the Cartesian components of the effective stress tensor

$\delta_{1}$ the Kronekr $\delta$

$\varepsilon_{i j} \quad$ the Cartesian components of the solid deformation tensor

\section{REFERENCES}

1. Sandbu, R.S.: 1968, Fluid Flow in Saturated Porous Elastic Media, Ph.D. Thesis, University of California at Berkeley, Berkeley, California

2. Sandhu, R.S., and Wilson, E.L.: 1969, "Finite Element Analysis of Seepage in Elastic Media", J. Engrg. Mech. Div, Am. Soc. Civ. Engrs., 95, pp.641-652.

3. Sandhu, R.S.: 1975, Variational Principles for Soil Consolidation, Report OSURF-357075-2to National Science Foundation, Dept. of Civil Engrg., The Ohio State University., Columbus, Ohio.

4. Sandhu, R.S.: 1976, "Variational Principles for Soil Consolidation", in Numerical Methods in Geomechanics, Proc., 2nd Intl. Conf. Numer. Methods in Geomech., Ed. C.S. Desai, Am. Soc. Civ. Engrs., l, pp.2040.

5. Ghaboussi, J., and Wilson, E.L.: 1973, "Flow of Compressible Fluid in Porous Elastic Media", Int, J. Num. Methods in Engrg., 5, pp.419-442.

6. Sendhu, R.S., Liu, $H_{\text {., }}$ and Singh, K.J.:1977, "Numerical Performance of Some Finite Element Schemes for Analysis of Seepage in Porous Elastic Media", Int. I. Num. Anal, Methods in Geomech., l, pp.177-194.

7. Sandhu, R.S, and Liu, H.:1979, "Analysis of Consolidation of Viscoelastic Soils", in Numerical Methods in Geomechanics, Proc., 3rd. Int. Conf.- Numer. Methods in Geomech., Ed. W. Wittke, A.A. Batkema, pp.1255-1263.

8. Prevost, J.H.: 1981, "Consolidation of Anelastic Porous Media", J. Engrg. Mech. Div, Am. Soc. Civ. Engrs., 107, pp. 169-186.

9. Booker, J.R.: 1973, "A Numerical Method for the Solution of Biot's Consolidation Theory", Quar. J. Mech. Appl. Math, XXVI, 4, pp.445-470.

10. Booker, J.R., and Small, J.C.: 1977, "Finite Element Analysis of Primary and Secondary Consolidation", Int. J. Solids Struct., 13, pp.137-149.

11. Carter, J.P., Booker, J.R, and Small, J.C.: 1979, "The Analysis of Elasto-Plastic Consolidation", Int. J. Num. Anal. Methods in Geomechanics, 3, pp.107-129.

12. Carter, J.P., Small, J.C., and Booker, J.R.: 1977, "A Theory of Finite Elastic Consolidation", Int. J. Solids Structures., 13, pp.467-478.

13. Siriwardane, J.H. and Desai, C.S.: 1981, "Two Numerical Schemes for Non-linear Consolidation", Int. J. Num. Methods in Engineering, 17, pp. 405-426.

14. Aboustit, B. L.: 1983, "Finite Element Investigations of Thermo-Elastic and ThernoPlastic Consolidation", Ph.D. Dissertation, The Ohio State University, Columbus, Ohio.

15. Aboustit, B. L., Advani, S.H. and Lee, J.K.: 1985, "Variational Principles and Finite Element Simulations for Thermo-Elastic Consolidation", International Journal for Numerical and Analytical Methods in Geomecharics, Vol 9, pp. 49-69.

16. Aboustit, B. L., Sandhu, R.S. and Hong, S.J.: 1984, "Response of Saturated Soils to Dyrlamic Loading", Annual Report, RF Project $763420 / 715107$, The Ohio State University Research Foundation.

17. Aboustit, B. L., Sandhu, R.S, and Hong, S.J.: 1985, "Analysis of Response of Saturated Systems Subjected to Dynamic Loading", Proceedings of the Second Symposium on the Interaction of Non-Nuclear Munitions with Structures, Panarna City Beach, Florida, pp. 364-370.

18. Korsawe, J. and Starke, G.: 2005, "A Least-Squares Mixed Finite Element Method for Biot's Consolidation Problem in Porous Media", SIAM J. Numer. Anal. Volume 43, Issue 1, pp. 318-339.

19. Ferronato, M., Castelletto, N. and Gambolati, G.:2010," A Fully Coupled 3-D Mixed Finite Element Model of Biot 
Consolidation", Joumal of Computational Physics, Volume 229, Issue 12, pp. 4813-4830.

20. Hwang, C.T., Morgenstern, N.R, and Murray, D.W.: 1971, "On Solution of Plane Strain Consolidation Problems by Finite Element Methods", Canadian Geotechnical I., 8, pp.109-118.

21. Smith, I.M., and Hobbs, R.: 1976, "Biot Analysis of Consolidation beneath Embankments", Geotechnique, 26, pp.149-171.

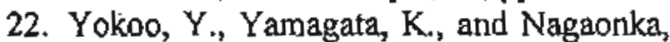
H.:1971, "Finite Element Method Applied to Biot's Consolidation Theory", Soils and Foundations, 11 , pp.29-46.

23. Booker, J.R, and Small, J.C.: 1975, "An Investigation of the Stability of Numerical Solution of Biot's Equations of Consolidation", Int. J. Solids Struet., 11, pp.907-917.
24. Vermeer, P.A., and Verruijt, A.: 1981, "An Accuracy Condition for Consolidation by Finite Elements", Int. J. Num. Aral. Methods in Geonecharics, 5, pp. 1-14.

25. A boustit, B. L., Sandhu, R.S. and Hong, S.J.: 1984, "An Evaluation of Finite Element Models For Soil Consolidation", Annual Report, RF Project 763420/715927, The Ohio State University Research Foundation.

26. Aboustit, B. L., Sandhu, R.S. and Hong, S.J.: 1985, "Finite Element Analysis of Flow and Deformation is Saturated Soils", Proceedings of the Second Symposium on the Interaction of Non-Nuclear Munitions with Structures, Parama City Beach, Florida, pp. 377-384. 